\title{
Correction to: Autism, Problematic Internet Use and Gaming Disorder: a Systematic Review
}

\author{
Alayna Murray ${ }^{1} \cdot$ Beatrix Koronczai $^{1} \cdot$ Orsolya Király $^{1} \cdot$ Mark D. Griffiths $^{2} \cdot$ Arlene Mannion $^{3} \cdot$ Geraldine Leader $^{3}$. \\ Zsolt Demetrovics ${ }^{1,4}$
}

Published online: 26 April 2021

(C) Springer Science+Business Media, LLC, part of Springer Nature 2021

\section{Correction to: Review Journal of Autism and Developmental Disorders https://doi.org/10.1007/s40489-021-00243-0}

The original article has been corrected. The fourth author affiliation must read as "Centre of Excellence in Responsible Gaming, University of Gibraltar, Gibraltar, Gibraltar" not "Centre of Excellence in Responsible Gaming, University of Gibraltar, Gibraltar, UK"

Publisher's Note Springer Nature remains neutral with regard to jurisdictional claims in published maps and institutional affiliations.

The online version of the original article can be found at https://doi.org/ 10.1007/s40489-021-00243-0

Zsolt Demetrovics

zsolt.demetrovics@unigib.edu.gi

1 Institute of Psychology, ELTE Eötvös Loránd University, Izabella utca 46, Budapest H-1064, Hungary

2 International Gaming Research Unit, Psychology Department, Nottingham Trent University, Nottingham, UK

3 Irish Centre for Autism and Neurodevelopmental Research (ICAN), School of Psychology, National University of Ireland,

Galway, Ireland

4 Centre of Excellence in Responsible Gaming, University of Gibraltar, Gibraltar, Gibraltar 